\title{
On the optimum distribution of enzyme feed in a cascade of CSTR's performing an enzyme-catalyzed reaction with deactivation
}

\author{
N.E. Moreira, F.X. Malcata
}

\begin{abstract}
A search for the optimum fractional distribution of an enzyme-rich stream to the various reactors of a cascade of CSTR's was implemented. A theoretical analysis, laid out in $q$ dimensionless form and based on the assumptions that the system is operated under steady state conditions, the enzyme undergoes first order deactivation, and the reaction catalyzed by the enzyme follows Michaelis-Menten kinetics, is reported. The objective function utilised is the minimisation of the overall volume of the cascade, and analytical expressions are obtained for the concentration of active enzyme and substrate in the outlet stream from each reactor. It is found that the best option is to add the whole enzyme-rich stream to the first reactor in the cascade irrespective of the operating and kinetic parameters of the system.
\end{abstract}

\begin{tabular}{|c|c|}
\hline \multicolumn{2}{|c|}{ List of symbols } \\
\hline$C_{E, i}$ & $\mathrm{~mol} \cdot \mathrm{m}^{-3}$ \\
\hline$C_{S, i}$ & $\mathrm{~mol} \cdot \mathrm{m}^{-3}$ \\
\hline$C_{S, i}^{*}$ & - \\
\hline$D a_{i}$ & - \\
\hline$\widehat{D} a_{i}$ & - \\
\hline$i$ & - \\
\hline j & - \\
\hline$k$ & $\mathrm{~s}^{-1}$ \\
\hline$K_{m}$ & $\mathrm{~mol} \cdot \mathrm{m}^{-3}$ \\
\hline$K_{m}^{\star}$ & - \\
\hline I & - \\
\hline$m$ & - \\
\hline$n$ & - \\
\hline
\end{tabular}

concentration of active enzyme in the $i$-th reactor concentration of substrate in the $i$-th reactor normalized concentration of substrate in the $i$-th reactor Damköhler number associated with the $i$-th reactor using as reference the volumetric flow rate of substrate to the first unit

Damköhler number associated with the $i$-th reactor using as reference the whole volumetric flow rate if all enzyme stream were added to the first unit dummy integer variable dummy integer variable deactivation constant Michaelis-Menten constant dimensionless Michaelis-Menten parameter dummy integer variable dummy integer variable dummy integer variable Dr. António Bernardino de Almeida, 4200 Porto, Portugal number of CSTRs in the cascade dummy integer variable dummy integer variable volumetric flow rate of substraterich stream dummy integer variable dummy integer variable dummy integer variable dummy integer variable dummy integer variable maximum rate of reaction at the $i$-th reactor holdup volume of the $i$-th reactor

fraction of overall enzyme added to the $i$-th reactor

Greek symbols

$\alpha_{i}$

$\beta$

ratio of overall volumetric flow rate of enzyme-rich stream to volumetric flow rate of substrate-rich stream to the first reactor

$\Theta_{i} \quad-\quad$ auxiliary function associated with the $i$-th reactor

$\begin{array}{ll}\tau_{i} & \mathbf{s} \\ \Xi & -\end{array}$ space time of the $i$-th reactor ratio of time constants associated with enzyme-catalyzed reaction and deactivation reaction

\section{1}

\section{Introduction}

The steady state operation of a cascade of continuous stirred tank reactors (CSTRs) performing a biochemical reaction catalyzed by an enzyme in soluble form requires a constant rate of addition of enzyme to the reactors. Although enzyme may be directly added in powdered form, mixing constraints usually indicate that the enzyme should be previously solubilized in a more or less concentrated aqueous buffer and hence should be added in liquid form rather than as a particulate solid.

A number of previous reports have dealt with minimization of the overall volume of isothermal cascades of micromixed CSTRs performing enzyme-catalyzed reactions in the absence of enzyme deactivation which follow irreversible MichaelisMenten kinetics [1], reversible Michaelis-Menten kinetics [2], multisited, highly cooperative Michaelis-Menten kinetics [3], and Ping Pong Bi Bi kinetics [4]; isothermal cascades of macromixed CSTRs performing enzyme-catalyzed reactions in 
the absence of enzyme deactivation which follow irreversible Michaelis-Menten kinetics [5]; isothermal cascades of micromixed CSTRs performing enzyme-catalyzed reactions with first order irreversible deactivation which follow irreversible Michaelis-Menten kinetics [6]; and nonisothermal cascades of micromixed CSTRs performing enzyme-catalyzed reactions with first order irreversible deactivation which follow irreversible Michaelis-Menten kinetics [7]. Comparison between the overall volume of a cascade made up of CSTRs designed using the minimum overall volume as optimisation criterion and the overall volume of a cascade containing CSTRs designed using equal sized reactors was also made for a given overall substrate conversion [8].

In all the aforementioned works one has hypothesized that the whole enzyme stream available should be added to the first reactor in the series in order to assume steady state operation, but no rationale was ever provided for such hypothesis from an applied point of view. However, if the rate of total enzyme to be added to a given cascade of CSTRs is previously fixed, then there might in principle be a fractional distribution of the overall enzyme-rich stream to the various reactors in the cascade that leads to the maximum value of the overall conversion of substrate or, equivalently, that leads to the minimum value of the overall reactor volume for a given conversion of substrate. The existence of such optimum is somewhat anticipated since there are two opposing factors: on the one hand, the conversion per unit active enzyme should increase when the enzyme molecules spend a longer mean residence time in the reactor cascade; on the other hand, adding the enzyme current earlier leads to higher dilutions of the reaction stream (with concomitant decreases in the rate of reaction) and increases the mean residence time of the enzyme in the reactor cascade (with a higher chance for deactivation of the enzyme).

It is the purpose of this communication to provide a mathematical framework that will allow decision on which distribution of the enzyme stream to the various CSTRs in a cascade should be selected if one seeks a minimum overall reactor volume able to achieve a given substrate conversion.

\section{2}

\section{Mathematical analysis}

Assume that an enzyme-catalyzed reaction takes place in a series of CSTR's with the feed of enzyme distributed by the various units as depicted in Fig. 1.

The steady-state mass balance to active enzyme in such a series of CSTR's may be written as:

$$
\begin{aligned}
& \frac{v_{\max , 1}}{v_{\max , 0}} \equiv \frac{C_{E, 1}}{C_{E, 0}}=\frac{\alpha_{1}}{1+\tau_{1} k+\alpha_{1}}, \\
& \frac{v_{\max , i}}{v_{\max , 0}} \equiv \frac{C_{E, i}}{C_{E, 0}}=\frac{\alpha_{i}+\left(1+\sum_{l=1}^{i-1} \alpha_{l}\right) \frac{C_{E, i-1}}{C_{E, 0}}}{1+\tau_{i} k+\sum_{j=1}^{i} \alpha_{j}}, i=2,3, \ldots, N-1 \\
& \frac{v_{\max , N}}{v_{\max , 0}} \equiv \frac{C_{E, N}}{C_{E, 0}}=\frac{\alpha_{N}+\left(1+\sum_{l=1}^{N-1} \alpha_{l}\right) \frac{C_{E, N-1}}{C_{E, 0}}}{1+\beta+\tau_{N} k}
\end{aligned}
$$

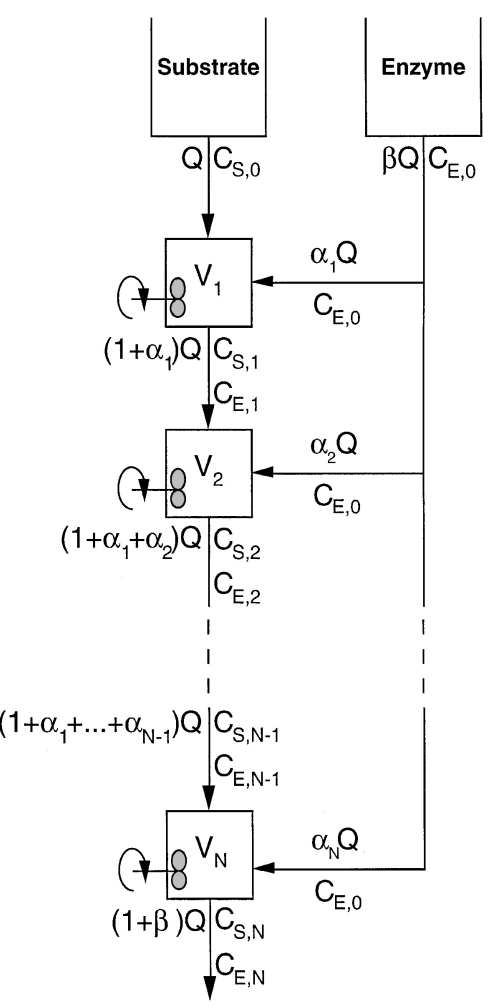

Fig. 1. Schematic diagram of the cascade of CSTR's with indication of the volumetric flow rates and concentrations of substrate and enzyme of each stream

where the enzyme is assumed to undergo thermal deactivation characterized by the first order kinetic constant $k$. The maximum rate of reaction at the $i$-th reactor is denoted by $v_{\max , i}$, the concentration of active enzyme in the same reactor by $C_{E, i}$, the fraction of the overall enzyme that is added to the inlet stream of the $i$-th reactor as $\alpha_{i}$, and the ratio of the overall volumetric flow rate of the enzyme-rich stream to the overall volumetric flow rate, $Q$, of the substrate-rich stream (which is all fed as inlet stream to the first reactor) as $\beta$. In Eq. (1), $\tau_{i} \equiv V_{i} / Q$ denotes the pseudo space time of the $i$-th reactor (i.e. the average time a volume element would spend in that reactor if the volumetric flow rate is equal to the flow rate of the inlet substrate stream to the first reactor in the series), where $V_{i}$ is the holdup volume of the $i$-th reactor.

Algebraic manipulation of Eq. (1) finally leads to:

$\frac{v_{\max , 1}}{v_{\max , 0}} \equiv \frac{C_{E, 1}}{C_{E, 0}}=\frac{\alpha_{1}}{1+\alpha_{1}+\Xi D a_{1}}$,

$\frac{v_{\max , 2}}{v_{\max , 0}} \equiv \frac{C_{E, 2}}{C_{E, 0}}=\frac{\alpha_{1}\left(1+\alpha_{1}\right)+\alpha_{2}\left(1+\alpha_{1}+\Xi D a_{1}\right)}{\left(1+\alpha_{1}+\Xi D a_{1}\right)\left(1+\alpha_{1}+\alpha_{2}+\Xi D a_{2}\right)}$,

$\frac{v_{\max , i}}{v_{\max , 0}} \equiv \frac{C_{E, i}}{C_{E, 0}}=\cdots$ 


$$
\ldots \frac{\left(\begin{array}{c}
\alpha_{i} \prod_{m=1}^{i-1}\left(1+\Xi D a_{m}+\sum_{n=1}^{m} \alpha_{n}\right)+\alpha_{1} \prod_{p=1}^{i-1}\left(1+\sum_{q=1}^{p} \alpha_{q}\right)+\cdots \\
\cdots \sum_{r=2}^{i-1} \alpha_{r}\left(\prod_{v=r}^{i-1}\left(1+\sum_{s=1}^{v} \alpha_{s}\right)\right)\left(\prod_{t=1}^{r-1}\left(1+\Xi D a_{t}+\sum_{u=1}^{t} \alpha_{u}\right)\right)
\end{array}\right)}{\prod_{j=1}^{i}\left(1+\Xi D a_{j}+\sum_{l=1}^{j} \alpha_{l}\right)},
$$

where $\Xi \equiv k . C_{S, 0} / v_{\max , i}$ denotes the ratio of the time constant associated with the enzyme-catalyzed reaction occurring in the $i$-th reactor (i.e. $C_{S, 0} / v_{\max , i}$, where $C_{S, 0}$ is the inlet substrate concentration to the first reactor) to the time constant associated with enzyme deactivation (i.e. $1 / k$ ), and where $D a_{i} \equiv v_{\max , i} \cdot \tau_{i} / C_{S, 0}$, or Damköhler number, is the ratio of the time constant associated with the forced convection through the $i$-th reactor (i.e. $\tau_{i}$ ) to the time constant associated with the enzyme-catalyzed reaction occurring in the $i$-th reactor (i.e. $\left.C_{S, 0} / v_{\max , i}\right)$.

The mass balance to the substrate in any reactor of the cascade under steady state conditions of operation is then given by:

$$
1-\left(1+\alpha_{1}\right) C_{S, 1}^{*}=\frac{D a_{1}\left(\frac{C_{E, 1}}{C_{E, 0}}\right) C_{S, 1}^{*}}{K_{m}^{*}+C_{S, 1}^{*}},
$$

$$
\left(1+\sum_{j=1}^{i-1} \alpha_{j}\right) C_{S, i-1}^{*}-\left(1+\sum_{l=1}^{i} \alpha_{l}\right) C_{S, i}^{*}=\frac{D a_{i}\left(\frac{C_{E, i}}{C_{E, 0}}\right) C_{S, i}^{*}}{K_{m}^{*}+C_{S, i}^{*}},
$$$$
i=2,3, \ldots, N-1 \text {, }
$$

$\left(1+\sum_{j=1}^{N-1} \alpha_{j}\right) C_{S, N-1}^{*}-(1+\beta) C_{S, N}^{\star}=\frac{D a_{N}\left(\frac{C_{E, N}}{C_{E, 0}}\right) C_{S, N}^{\star}}{K_{m}^{\star}+C_{S, N}^{*}}$,

where the substrate was assumed to undergo an enzymecatalyzed reaction described by Michaelis-Menten kinetics, where the normalized concentration of substrate in the $i$-th reactor is defined as $C_{S, i}^{*} \equiv C_{S, i} / C_{S, 0}\left(C_{S, i}\right.$ is the substrate concentration in the $i$-th reactor), and where the dimensionless parameter is defined as $K_{m}^{\star} \equiv K_{m} / C_{S, 0}$ (the Michaelis-Menten constant is denoted as $K_{m}$ ).

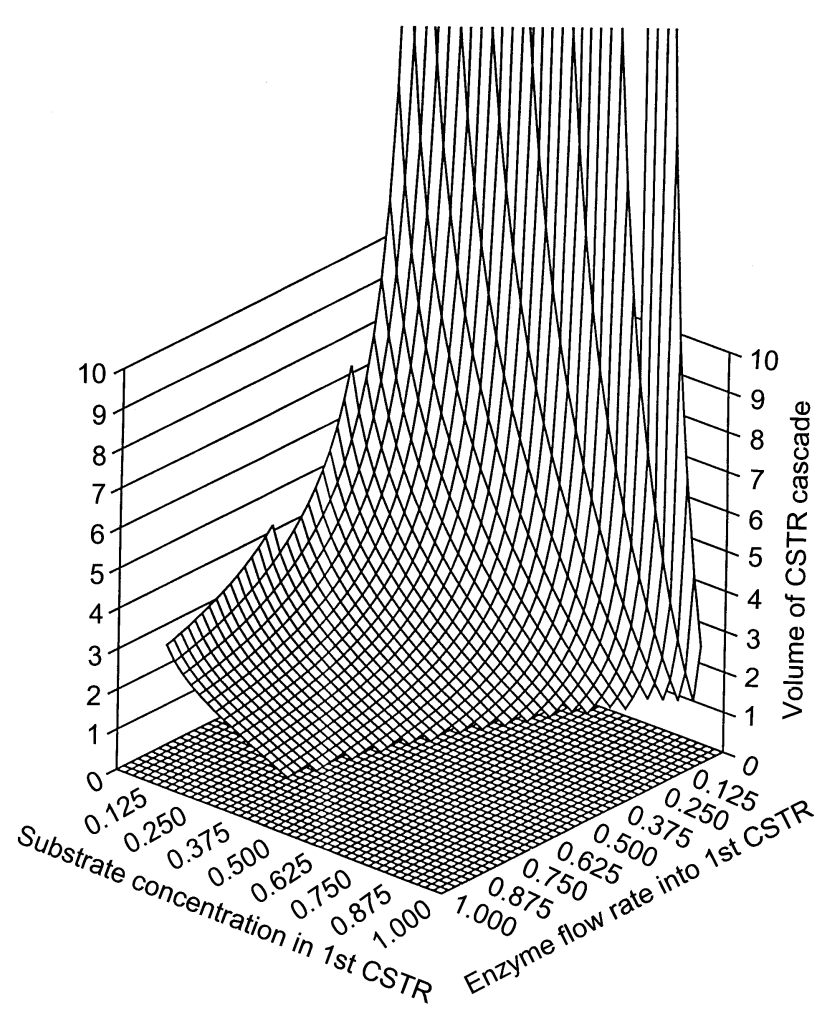

Fig. 2. Plot of the volume of the CSTR cascade composed of two units $\left(D a_{1}+D a_{2}\right)$ as a function of the enzyme flow rate into the first CSTR $\left(\alpha_{1}\right)$ and the substrate concentration in the first $\operatorname{CSTR}\left(C_{S, 1}^{*}\right)$ for $K_{m}^{*}=0.1$, $\Xi=0.1, \beta=1$, and $C_{S, 2}^{*}=0.1$

Eliminating $C_{E, i} / C_{E, 0}$ in Eq. (3) for every $i$ using the corresponding forms of Eq. (2), solving each of the resulting equations for $D a_{j}$, and then using the value of each $D a_{i}$ in the $(i+1)$-th equation, one obtains, after some algebraic manipulation:

$D a_{1}=\frac{\left(1+\alpha_{1}\right) \frac{\left(K_{m}^{*}+C_{S, 1}^{*}\right)\left(1-\left(1+\alpha_{1}\right) C_{S, 1}^{*}\right)}{C_{S, 1}^{*}}}{\alpha_{1}-\Xi \frac{\left(K_{m}^{*}+C_{S, 1}^{*}\right)\left(1-\left(1+\alpha_{1}\right) C_{S, 1}^{*}\right)}{C_{S, 1}^{*}}}$,

and

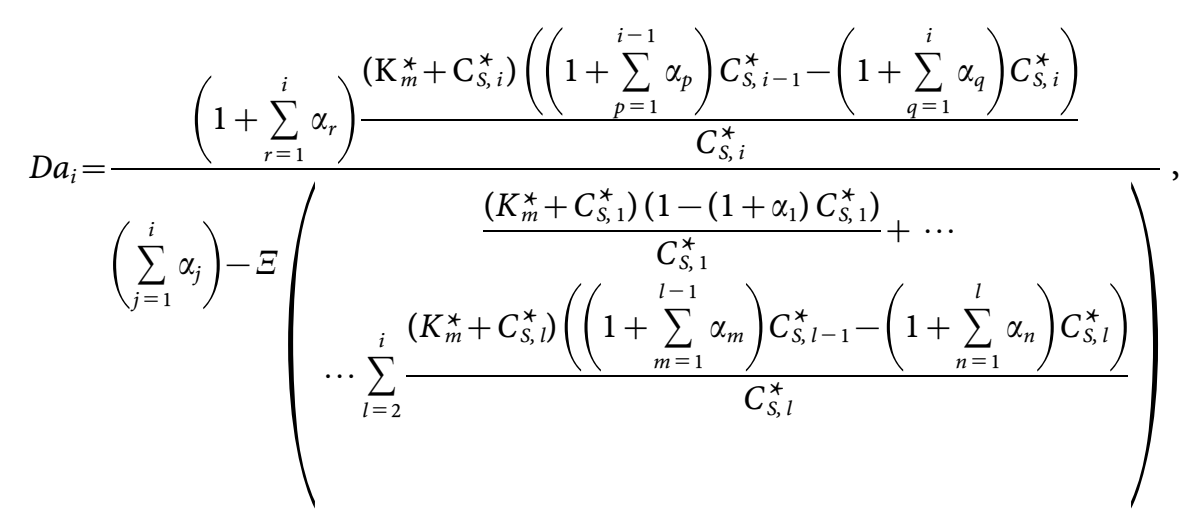$$
i=2,3, \ldots, N
$$ 
The variation of the overall Damköhler number for a cascade of two CSTRs versus $\alpha_{1}$ and $C_{S, 1}^{*}$ is depicted in Fig. 2 for a typical combination of parameter values with physical and technological significance.

Rewriting Eq. (4) as:

$D a_{1}=\frac{\left(1+\alpha_{1}\right) \Theta_{1}\left\{K_{m}^{*} ; C_{S, 1}^{*}, \alpha_{1}\right\}}{\alpha_{1}-\Xi \Theta_{1}}$,

and

The expressions obtained from combination, for several values of $N$, of Eqs. (5) and (7) were, following algebraic rearrangement, used in comprehensive trial-and-error searches of solutions using factorial grids in $\alpha_{1}, \alpha_{2}, \ldots, \alpha_{N-1}$ for several values of $K_{m}^{*}, \beta, \Xi$, and $C_{S, i}^{*}(i=1,2, \ldots, N)$, on the one hand, and using factorial grids in $C_{S, 1}^{*}, C_{S, 2}^{*}, \ldots, C_{S, N-1}^{*}$ for several values of $K_{m}^{*}, \beta, \Xi, \alpha_{i}(i=1,2, \ldots, N)$ and $C_{S, N}^{\star}$, on the other.

where the auxiliary functions are defined as:

$\Theta_{1}\left\{K_{m}^{*} ; C_{S, 1}^{*}, \alpha_{1}\right\}=\frac{\left(K_{m}^{*}+C_{S, 1}^{*}\right)\left(1-\left(1+\alpha_{1}\right) C_{S, 1}^{*}\right)}{C_{S, 1}^{*}}$,

and

$\Theta_{l}\left\{K_{m}^{*} ; C_{S, l-1}^{*}, C_{S, l}^{*}, \alpha_{l-1}, \alpha_{i}\right\}=\frac{\left(K_{m}^{*}+C_{S, l}^{*}\right)\left(\left(1+\sum_{m=1}^{l-1} \alpha_{m}\right) C_{S, l-1}^{*}-\left(1+\sum_{n=1}^{l} \alpha_{n}\right) C_{S, l}^{*}\right)}{C_{S, l}^{*}}$

$$
l=2,3, \ldots, N,
$$

respectively. If one sets as the objective function minimisation of the overall volume of the cascade of CSTR's, then the necessary conditions for such optimum to exist are given by:

$\left\{\frac{\partial\left(\sum_{i=1}^{N} D a_{i}\right)}{\partial C_{S, j}^{*}}\right\}_{\Xi, \beta, K_{m}^{\star}, C_{S, l \neq j}^{*}, \alpha_{n}}=0, j=1,2, \ldots, N-1$,

and

$\left\{\frac{\partial\left(\sum_{i=1}^{N} D a_{i}\right)}{\partial \alpha_{j}}\right\}_{\Xi, \beta, K_{m}^{*}, C_{S, l}^{*}, \alpha_{n \neq j}}=0, j=1,2, \ldots, N-1$,
When implementing the aforementioned searches special care was exercised in order to prevent violation of physical restrictions; these restrictions arise from dilution considerations, viz:

$$
\left(1+\sum_{p=1}^{i-1} \alpha_{p}\right) C_{S, i-1}^{*} \geqslant\left(1+\sum_{q=1}^{i} \alpha_{q}\right) C_{S, i}^{*} \text { for } i=1,2, \ldots, N
$$

or, after consideration of Eq. (5), by:

$$
\sum_{i=j}^{N}\left(\left\{\frac{\partial D a_{i}}{\partial \Theta_{j}}\right\}_{\Xi, \alpha_{n}}\left\{\frac{\partial \Theta_{j}}{\partial C_{S, j}^{*}}\right\}_{K_{m}^{*}, C_{S, l \neq j}^{*}, \alpha_{n}}+\left\{\frac{\partial D a_{i}}{\partial \Theta_{j+1}}\right\}_{\Xi, \alpha_{n}}\left\{\frac{\partial \Theta_{j+1}}{\partial C_{S, j}^{*}}\right\}_{K_{m}^{*}, C_{S, l \neq j}^{*}, \alpha_{n}}\right)=0
$$

$$
j=1,2, \ldots, N-1 \text {, }
$$

$\sum_{i=j}^{N}\left\{\left\{\frac{\partial D a_{i}}{\partial \alpha_{j}}\right\}_{\Xi, \alpha_{n \neq j}, \theta_{m}}^{\text {and }}+\left\{\frac{\partial D a_{i}}{\partial \Theta_{j}}\right\}_{\Xi, \alpha_{n}}\left\{\frac{\partial \Theta_{j}}{\partial \alpha_{j}}\right\}_{K_{m}^{\star}, C_{, l,}^{*}, \alpha_{n \neq j}}+\left\{\frac{\partial D a_{i}}{\partial \Theta_{j+1}}\right\}_{\Xi, \alpha_{n}}\left\{\frac{\partial \Theta_{j+1}}{\partial \alpha_{j}}\right\}_{K_{m}^{*}, C_{s, l}^{*}, \alpha_{n \neq j}}\right)=0$

$j=1,2, \ldots, N-1$. 
from deactivation considerations, viz.:
$\left(\sum_{j=1}^{i} \alpha_{j}\right)>\Xi \sum_{l=1}^{i} \frac{\left(K_{m}^{*}+C_{S, l}^{*}\right)\left(\left(1+\sum_{m=1}^{l-1} \alpha_{m}\right) C_{S, l-1}^{*}-\left(1+\sum_{n=1}^{l} \alpha_{n}\right) C_{S, l}^{*}\right)}{C_{S, l}^{*}}$

for $i=1,2, \ldots, N$, and from flow rate considerations, viz. $0 \leqslant \alpha_{i} \leqslant \beta$ for $i=1,2, \ldots, N$ and $\sum_{i=1}^{N} \alpha_{i}=\beta$. When the $C_{S, i}^{*}$ are fixed then it is in general possible to find solutions for Eq. (7.2); these solutions correspond to the distribution of enzyme feed through the cascade that yields a local minimum for the overall reactor volume for a given conversion in each reactor. By a similar token, when the $\alpha_{i}$ are fixed then it is in general possible to find solutions for Eq. (7.1); these solutions correspond to the profile of substrate concentrations through the cascade that yields a local minimum for the overall reactor volume for a given distribution of enzyme feed and for a given overall conversion. The former loci and the latter loci do, however, consistently intersect on a restriction, i.e. the minimum of the minima lies always on $\alpha_{1}=\beta$ and $\alpha_{2}=\alpha_{3}=\cdots=\alpha_{N}=0$. This interesting observation means that all enzyme should be added to the first reactor in the cascade, in which case the following asymptotic form of Eq. (4) can be written:

$$
\begin{aligned}
& D a_{1}=\frac{(1+\beta) \frac{\left(K_{m}^{*}+C_{S, 1}^{*}\right)\left(1-C_{S, 1}^{*}\right)}{C_{S, 1}^{*}}}{\beta-\Xi \frac{\left(K_{m}^{*}+C_{S, 1}^{*}\right)\left(1-C_{S, 1}^{*}\right)}{C_{S, 1}^{*}}}, \\
& D a_{i}=\frac{(1+\beta) \frac{\left(K_{m}^{*}+C_{S, i}^{*}\right)\left(C_{S, i-1}^{*}-C_{S, i}^{*}\right)}{C_{S, i}^{*}}}{\beta-\Xi\left(\frac{\left(K_{m}^{*}+C_{S, 1}^{*}\right)\left(1-C_{S, 1}^{*}\right)}{C_{S, 1}^{*}}+\sum_{l=2}^{i} \frac{\left(K_{m}^{*}+C_{S, l}^{*}\right)\left(C_{S, l-1}^{*}-C_{S, 1}^{*}\right)}{C_{S, l}^{*}}\right)}, \\
& i=2,3, \ldots, N \text {. }
\end{aligned}
$$

numerical evidence was given for the existence of a maximum overall conversion of substrate when all enzyme is fed to the first reactor in the series (which accounts for the physical constraint associated with deactivation introduced above), whereas the actual optima of intermediate substrate concentrations in such asymptotic situation that lead to the minimum overall volume for each given conversion is provided elsewhere [6].

The major conclusion reached in this research work is important from a practical point of view because it provides the heuristic rule that all enzyme should be fed to the first unit of a cascade of CSTRs irrespective of how dilute such enzyme is added. Such conclusion is not, however, of a trivial nature. In fact, it is commonly accepted (e.g. [10]) that series combinations of given reactors always provide higher reaction extents than parallel combinations, even if heuristics rule that only outlet streams with the same composition should be mixed. Since the type of reactor combinations suggested, series with respect to the substrate streams but is parallel with respect to the enzyme streams (see Fig. 1), then it would be anticipated, from the point of view of the enzymatic reaction, that such intermediate configuration would lead to lower conversions than feeding all substrate and all enzyme to the
Since in this situation all enzyme is added to the first reactor in the series (or, equivalently, to the inlet stream prior to entrance in the first reactor), then the volumetric flow rate through the reactor cascade (i.e. $(1+\beta) Q$ ) is constant. If this flow rate is used in the definition of the Damköhler number, and if the simplified counterparts of Eq. (2) are employed, then Eq. (8) becomes:

$$
\widehat{D} a_{1}=\frac{\frac{\left(K_{m}^{*}+C_{S, 1}^{*}\right)\left(1-C_{S, 1}^{*}\right)}{C_{S, 1}^{*}}}{1-\Xi \frac{\left(K_{m}^{*}+C_{S, 1}^{*}\right)\left(1-C_{S, 1}^{*}\right)}{C_{S, 1}^{*}}},
$$

$$
\widehat{D} a_{i}=\frac{\frac{\left(K_{m}^{*}+C_{S, i}^{*}\right)\left(C_{S, i-1}^{*}-C_{S, i}^{*}\right)}{C_{S, i}^{*}}}{1-\Xi\left(\frac{\left(K_{m}^{*}+C_{S, 1}^{*}\right)\left(1-C_{S, 1}^{*}\right)}{C_{S, 1}^{*}}+\sum_{l=2}^{i} \frac{\left(K_{m}^{*}+C_{S, l}^{*}\right)\left(C_{S, l-1}^{*}-C_{S, l}^{*}\right)}{C_{S, l}^{*}}\right)},
$$

very first reactor in the series. However, deactivation of enzyme exists, and for this reaction the same heuristic rule applies, i.e. the highest extent of enzyme deactivation is expected to take place when all enzyme is fed to the first reactor in the series. Therefore, a compromise should in principle be reached between such two opposing factors, which means that the possibility of feeding the enzyme fractionally to the various units would deserve further consideration. The conclusion obtained thus suggests that the increase in the deactivation extent brought about by feeding all enzyme to the first reactor and the decrease in the enzymatic conversion brought about by diluting the substrate a form which is consistent with results reported elsewhere [9] provided that $\widehat{D} a_{i} \equiv v_{\max , i} \cdot V_{i} /\left((1+\beta) Q C_{S, 0}\right)$; in that report stream right from the first reactor (or, equivalently, by decreasing the volumetric flow rate of substrate through the 
cascade right from the first reactor) are always overbalanced by the decrease in the deactivation extent brought about by decreasing the enzyme concentration when it is diluted with the substrate stream right in the first reactor (or, equivalently, by decreasing the volumetric flow rate of enzyme through the cascade right from the first reactor) and by the increase in the enzymatic conversion brought about by mixing the substrate with all the enzyme available right in the first unit.

\section{References}

1. Luyben, K.C.; Tramper, J.: Optimal design for continuous stirred tank reactors in series using Michaelis-Menten kinetics. Biotechnol. Bioeng. 24 (1982) 1217-1220

2. Malcata, F.X.; Cameron, D.C.: Optimal design of a series of CSTR's performing reversible reactions catalyzed by soluble enzymes: a theoretical study. Biocatalysis 5 (1992) 233-248

3. Malcata, F.X.: CSTR's in biochemical reactions - an optimization problem. Chem. Eng. Ed. 23 (1989) 112-115, 128

4. Malcata, F.X.: Optimal design on an economic basis for continuous stirred tank reactors in series using Michaelis-Menten kinetics for ping-pong reactions. Can. J. Chem. Eng. 66 (1988) 168-172

5. Malcata, F.X.: The effect of the level of micromixing on the optimal design of CSTR's performing Michaelis-Menten reactions. Can. J. Chem. Eng. 68 (1989) 330-336

6. Lopes, T.I.; Malcata, F.X.: Optimal design of a series of CSTR's for biochemical reactions in the presence of enzyme deactivation. J. Chem. Eng. Japan 26 (1993) 94-98

7. Paiva, A.L.; Malcata, F.X.: Optimal temperature and concentration profiles in a cascade of CSTR's performing Michaelis-Menten reactions with first order enzyme deactivation. Bioprocess Eng. 9 (1993) 77-82

8. Malcata, F.X.: To what extent is a constant volume design worse than a minimum volume design for a series of CSTR's? Bioprocess Eng. 13 (1995) 105-107

9. Malcata, F.X.: On the maximum conversion of substrate during biochemical reactions performed by a series of CSTRs in the presence of enzyme deactivation. J. Chem. Eng. Japan 23 (1990), 372-375

10. Hill, C.G.: An Introduction to Chemical Engineering Kinetics and Reactor Design. Wiley, New York (1977) 